\title{
Extracting representative structures from protein conformational ensembles
}

Alberto Perez ${ }^{1}$, Arijit Roy ${ }^{1}$, Koushik Kasavajhala ${ }^{1}$, Amy Wagaman $^{3}$, Ken A. Dill ${ }^{1,2}$, Justin L. MacCallum, ${ }^{1 *}$

${ }^{1}$ Laufer Center for Physical and Quantitative Biology, Stony Brook University

${ }^{2}$ Departments of Physics and Chemistry, Stony Brook University

${ }^{3}$ Department of Mathematics, Amherst College

* To whom correspondence should be addressed. Email: justin.maccallum@me.com

\begin{abstract}
A large number of methods generate conformational ensembles of biomolecules. Selecting one structure to be representative of the whole ensemble is often performed and is usually done by clustering and selecting the structure closest to the average of the most populated cluster. We find that this structure is not necessarily the best representation of the cluster and present here two computationally inexpensive averaging protocols that can systematically provide better representations of the system, which can be more directly compared with structures from X-ray crystallography. In practice, errors in the underlying force fields appear to limit the maximum improvement of averaging methods.
\end{abstract}

\section{Introduction}

There are a variety of methods that generate distributions of protein conformations. Examples include molecular dynamics simulations [ref], structure determination by refinement of nuclear magnetic resonance (NMR) data [ref], and computational tools for protein structure prediction [ref]. We often want to compare these distributions with the ensemble average structure from X-ray crystallography. The question we address in this paper is: How do we choose or generate a structure that is representative of the conformational ensemble, which can be directly compared with the average structure from X-ray crystallography? 


\section{Conformational entropy ensures that few structures from an ensemble are close to the average}

To see the difficulty of this problem, let us consider an ensemble generated based on the information from the X-ray crystal structure, which provides an explicit model for the mean conformation and associated fluctuations. The coordinates from the crystal structure give the mean position for each atom; each atom undergoes independent, isotropic, Gaussian fluctuations with a variance related to the crystallographic B-factor. We can generate conformations from this distribution and ask how close they are to the mean structure.

Figure 1 shows that structures from this distribution are almost never very close to the mean-even though the most likely position for each atom is the mean position and the most likely single structure is the mean structure. Why does this happen? The force at work is conformational entropy. There are simply far more conformations with high RMSD than with low RMSD [ref kuntz] and thermal motions are constantly pushing atoms away from their average positions. Even though each atom is most likely to be at its mean position, the probability of having all atoms simultaneously close to their mean position is negligible.

This example illustrates the difficulty of comparing any structure generated from an ensemble with the average structure from X-ray crystallography. In this case, we have a perfect "energy function" and can generate an almost arbitrarily large sample of conformations, but the chance of generating a structure very close to the average is extremely small. This effect becomes more pronounced the more flexible the protein is and the broader the conformational ensemble becomes, because the average RMSD is directly related to the size of the atomic fluctuations (see caption of Figure 1). 


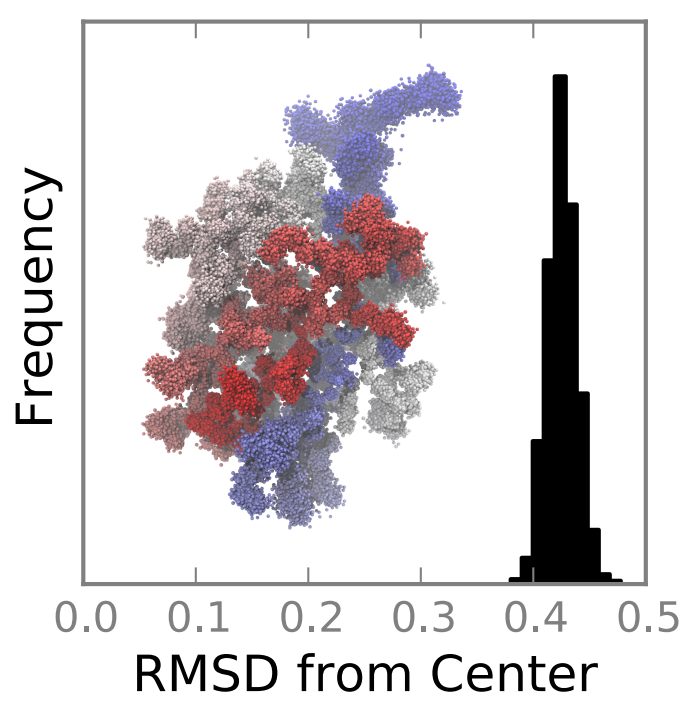

Figure 1: Conformational entropy pushes conformations away from the crystal structure. The inset shows 1000 conformations of ubiquitin (pdb: $1 \mathrm{UBQ}$, chain is colored red to blue from $\mathrm{N}$ - to C-terminus) generated based on the coordinates and B-factors from X-ray crystallography. The overall all-atom RMSD is tightly peaked around $0.42 \AA$, which is related to the crystallographic B-factors $(\langle R M S D\rangle=$ $\left.\left\langle\frac{B}{8 \pi^{2}}\right\rangle^{0.5}\right)$.

However, there is enough information in the ensemble shown in Figure 1 to accurately estimate the average structure. In the case of this simple independent, isotropic, Gaussian model, by simply taking the mean position of each atom-which we refer to as the Cartesian Average or CartAvg - we can obtain an accurate estimate of the average structure. However, non-linearities and correlations between atoms (described in the next two sections) can complicate matters and lead to CartAvg to produce sub-optimal estimates, which motivated us to develop a graph theoretic method called NetAvg.

In this paper, we will compare CartAvg and NetAvg with the common practice of choosing the structure closest to the center of the ensemble, called the medoid. We will examine two types of datasets: (1) cases with ideal distributions, which have been constructed such that the ensemble is guaranteed to fluctuate around the experimental structure; and (2) real-world distributions where the energy function is not perfect and thus the ensemble might not be centered around the experimental structure.

\section{Some atoms have non-linear distributions}

In the previous example, the distribution of each atom was an isotropic Gaussian centered on the mean position. However, not all atomic distributions are so simple. 
Some atoms have more complex non-linear distributions-particularly atoms in surface sidechains and in loops. For example, in a surface lysine residue, the length between the $\alpha$-carbon and the terminal nitrogen is relatively constant, but the angular degrees of freedom are much more flexible. This leads to a dome-shaped distribution of the nitrogen atom (Figure 2A). Taking the mean of such a distribution produces an average position that does not lie within the cloud of positions that were actually sampled (Figure 2B). The mean position is artificially "compressed" towards the point of rotation.

A better "average" position - which is not really an average, but rather a "representative" position - would be one that lies near the most densely populated region of the point cloud. We have developed a graph theoretic method called NetAvg that attempts to capture better representative structures from such nonlinear distributions. We defer description of the algorithm of NetAvg until the Methods section. 
A
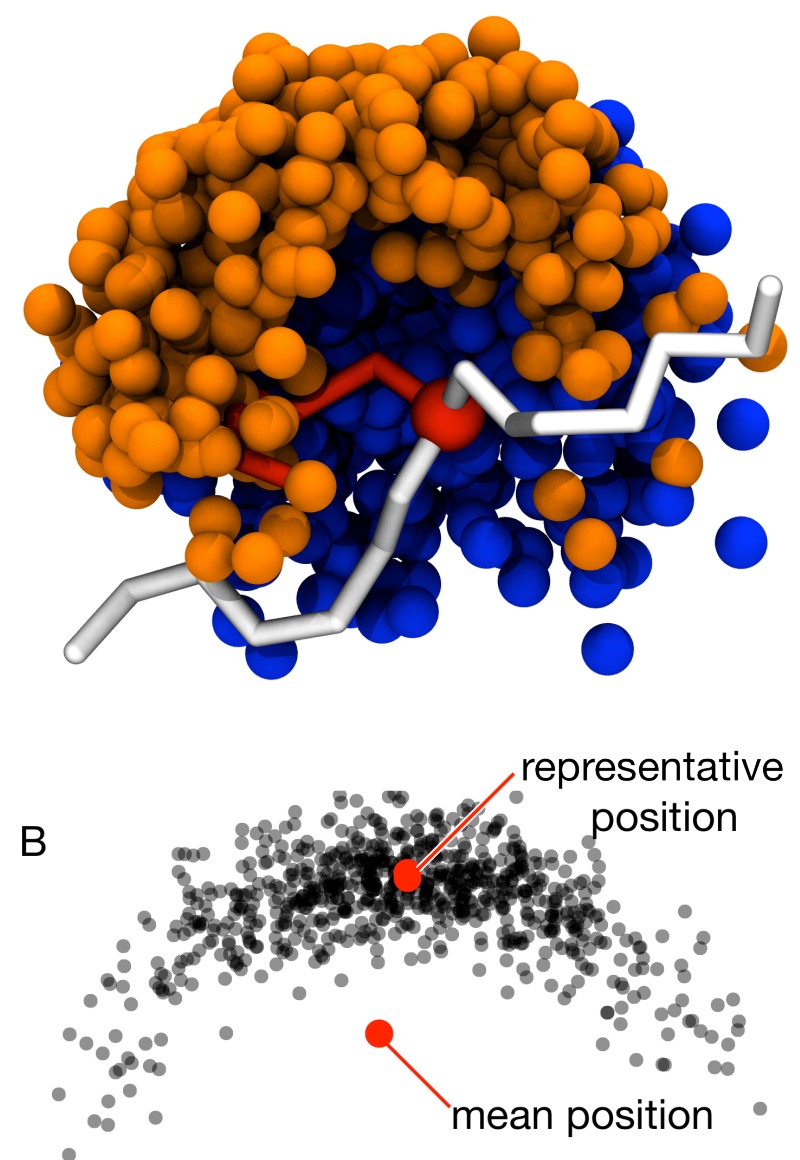

D

$+$
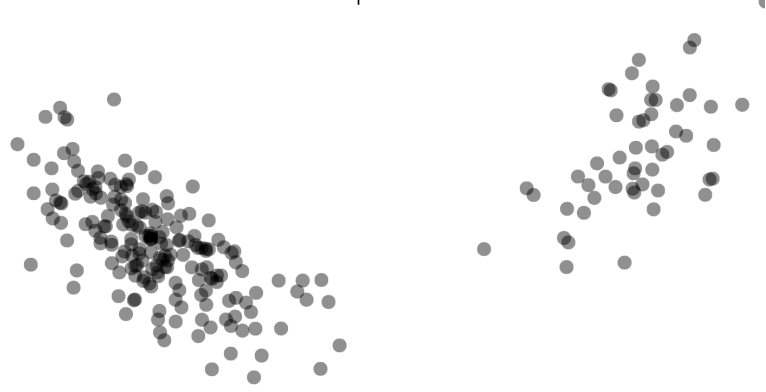

Figure 2: Some atoms have curved distributions, where simply taking the mean leads to an unrealistic average position. (A) Highly non-linear distribution of positions for the terminal nitrogen of a lysine sidechain. The protein backbone is white and the lysine sidechain is red. Each sphere represents a position of the terminal nitrogen group from the ensemble. The spheres are colored blue or orange to emphasize the curved shape. (B) Schematic 2D illustration of a complex non-linear distribution of atom positions. The mean position lies in a region of space that is never sampled. A better representative position is shown.

Ignoring correlations between atoms leads to unphysical structures 
With NetAvg and CartAvg, we are considering the position of one atom at a time and trying to "project" towards a more central structure that is not in the ensemble. When determining the average position with either method, one of the major approximations we are making is to ignore correlations between atom positions. We assume that the average position of each atom can be determined independently, ignoring the position of other atoms.

This often turns out to be a bad approximation. Physical forces like bonds and steric repulsion lead to strong correlations between the positions of atoms that are located close together in space. By ignoring these correlations when computing the average, we often produce models that appear to violate these physical forces and have unrealistic bond geometry and unphysical steric clashes. For example, the rigid chemical structure of a phenylalanine ring introduces coupling between atoms on opposite sides of the ring. The averaging methods ignore these correlations and can lead to unphysical structures (Figure 3, Unphysical Average).

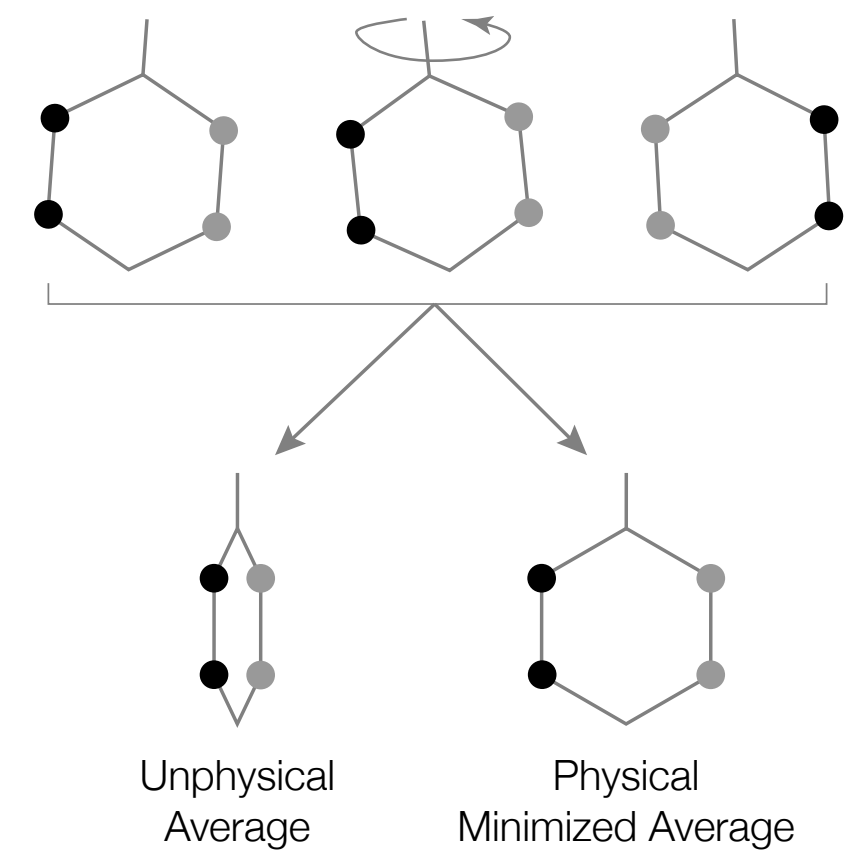

Figure 3: Ignoring correlations between atoms leads to unphysical average structures. At the top are three conformations of a phenylalanine ring undergoing rotation. Ignoring correlations leads to an unphysical average structure. Instead, a targeted minimization procedure can be used (see Methods) that produces a physically realistic conformation.

For NetAvg and CartAvg, we do not deal with this problem of correlations producing unphysical structures directly. Instead, we compute the raw average structure ignoring correlations and then use a targeted energy minimization procedure (see Methods) to produce a physically plausible structure that is close to the raw average structure (Figure 3, Physical Minimized Average). 


\section{Methods}

Scripts for performing the following calculations are available with an open source license (http://www.github.com/laufercenter/NetAvg).

\section{Iterative superposition}

As a first step, each conformation from the ensemble is iteratively superposed onto the mean conformation until convergence. This ensures that all conformations are located in a single coordinate system where the maximum amount of translational and rotational motion has been removed. This is accomplished using the "interpose" function from the "PDBEnsemble" class of Prody [prody] with the default tolerance of $10^{-4} \AA$.

\section{Medoid: Representing the ensemble with the member closest to the Cartesian} average

The medoid structure is the structure from the ensemble that is closest to the mean structure. The medoid is commonly used as a representative structure from a cluster or ensemble [refs]. We compute the medoid structure by first finding the average structure of the cluster by iterative superposition (described above) and then selecting the member of the ensemble with the smallest RMSD to the average.

\section{CartAvg: Representing the ensemble with a physically plausible estimate based} on the Cartesian average

While the medoid structure is always a structure that was present in the initial ensemble, the CartAvg or Cartesian average structure is an attempt to "project" towards the center of the ensemble.

The CartAvg structure is obtained by first iteratively superposing all structures from the ensemble onto the mean structure until convergence (described above). Next, the average structure is computed by considering each atom independently. The $\mathrm{x}$, $\mathrm{y}$, and $\mathrm{z}$-coordinates are simply the mean $\mathrm{x}, \mathrm{y}$, and $\mathrm{z}$-coordinates of that atom in the ensemble. Thus, CartAvg not only ignores correlations between atoms, it also ignores correlations between the $\mathrm{x}, \mathrm{y}$, and $\mathrm{z}$-coordinates for each atom.

The coordinates of the mean structure are then used as a target for energy minimization (described below) to produce a physically meaningful structure that is close to the Cartesian average. 
NetAvg: Representing the ensemble using a physically plausible estimate based on a non-linear network-based average

Physical forces acting on each atom can introduce correlations, not only between atoms, but also between the $\mathrm{x}-, \mathrm{y}-$, and $\mathrm{z}$-coordinates of each atom. As shown in Figure 2, if an atom has a non-linear distribution, the mean position will often lie in a region of space that is never sampled during the simulation. The NetAvg attempts to correct this by using a nonlinear graph-based approach that tries to choose an average position that lies within the cloud of positions sampled by each atom.

NetAvg still ignores correlations between atoms. However, unlike CartAvg, it explicitly includes correlations between the $\mathrm{x}^{-}, \mathrm{y}-$, and $\mathrm{z}$-coordinates of each atom, which allows it to capture non-linearities in the atomic distributions.

The algorithm of NetAvg begins by iterative superposition of all structures to the mean structure (described above). Next, each atom is considered individually:

1. Create a graph where each position sampled by the atom is a node. There will be $N_{\text {frames }}$ nodes.

2. Compute the distance between all pairs of nodes as the Euclidean distance between the corresponding atom positions.

3. Connect each node to its nearest $\mathrm{k}$ neighbors with directed edges, where $\mathrm{k}$ is a parameter of the algorithm. We use $k=32$ in this work.

4. If two nodes are connected by edges in both directions, replace these edges with an undirected edge. Otherwise, remove any connections between the two nodes.

5. Find the largest connected component of the graph.

6. Find the node in the largest connected component with the highest eigenvector centrality [ref].

7. Compute the average position for the atom as the mean position of this node and all of the nodes connected to it (up to $\mathrm{k}$ other nodes).

The graph is built by connecting each node with its k-nearest neighbors. We found a value of $k=32$ worked well for a variety of systems (data not shown). If $\mathrm{k}$ becomes too small, the resulting averages become very noisy and unreliable. As $\mathrm{k}$ becomes large, all nodes become connected and the resulting average is the same as the CartAvg.

The eigenvector centrality is a measure of the importance of a node within a network and is related to Google's PageRank method for ranking web pages [ref]. The eigenvector centrality of node $i$ is the i'th component of the eigenvector corresponding to the largest eigenvalue of the following equation:

$$
A x=\lambda x
$$


where $\mathrm{A}$ is the adjacency matrix of the graph.

As with CartAvg, the structure obtained using this procedure is then used as a target during energy minimization (next section).

\section{Targeted Energy Minimization: Obtaining physically reasonable representative}

\section{structures}

In order to produce physically reasonable average models using NetAvg or CartAvg, we combine these averaging methods with molecular dynamics-based energy minimization. Instead of reporting the average structure directly, we first select the structure from the ensemble that is closest to the average from NetAvg or CartAvg. Next, we perform an energy minimization to pull the selected structure towards the average structure in the presence of a physical force field, as described below. We found that minimizing the structure in this way worked better than trying to directly minimize the average structure, which often led the minimizer to get stuck due to the distorted initial geometry.

All minimizations were carried out using the GROMACS [gromacs] software package, version 4.5.5. The protein was modeled using the FF99SB-ILDN force field [ildn]. Water was modeled implicitly using the generalized Born model of Onufriev, Bashford, and Case [obc]. The hydrophobic effect was approximated using the ACE approximation [ace]. The selected model from the ensemble was pulled towards the average model using Cartesian position restraints on all heavy atoms with a force constant of $1000 \mathrm{~kJ} / \mathrm{mol} / \mathrm{nm}^{2}$. The energy was minimized with 1000 steps of steepest decent, followed by 1000 steps of l-BFGS [bfgs].

\section{Assessing Success}

We use four measures to determine if the averaging protocols have been successful: heavy-atom RMSD, GDT-HA, CAD-score, and MolProbity (all defined below).

The heavy-atom root mean squared deviation (RMSD) measures the average deviation between the positions of the non-hydrogen atoms in the model with those in the experimental structure after rigid body superposition to minimize the deviation [rmsd]. Some residues contain chemically identical atoms related by internal rotations-Phe: CD1-CD2 and CE1-CE2; Tyr: CD1-CD2 and CE1-CE2; Asp: OD1-OD2; Glu: OE1-OE2; Leu: CD1-CD2; Val: CG1-CG2. These atoms were excluded from the all-atom RMSD calculation.

The high accuracy version of the global distance test (GDT-HA) measures how accurately the $\mathrm{C}^{\alpha}$ atoms are positioned [gdt]. GDT-HA ranges from 0 (bad) to 100 
(good) and can be understood approximately as the percentage of $\mathrm{C}^{\alpha}$ atoms that are positioned correctly

The contact area difference (CAD) score is a recently developed all-atom measure of model quality [cadscore], which is based on comparing contact areas between residues in the experimental and model structures. CAD-score is an all-atom measure that is sensitive to backbone and sidechain positioning. CAD-score ranges from 0 (bad) to 1.0 (good).

MolProbity [molprobity] is the only non-native-centric score used in our assessment of success. Rather than comparing the model with the experimental structure, MolProbity assesses how well the model matches physical constraints derived from examining high-resolution X-ray crystal structures. Lower scores indicate better, more physically plausible models.

We assessed if the differences between the different averaging methods were significant by performing the two-sided sign test. We consider value of $p<0.01$ to be significant. The significance results are detailed in Supplementary Table 1.

\section{Generation of ideal ensembles}

In order to test the ability of the different algorithms to compute average models close to the crystal structure, we used two idealized simple models that we call Gauss and Gō. By construction, these models have the crystal structure at the "center" and we are able to compare performance of the different averaging schemes without worrying about issues of force field accuracy and sampling quality. We used three simple proteins with a mixture of secondary structures as test systems: Protein A (pdb: 1bdd), Protein G (pdb: 2gb1), and Ubiquitin (pdb: 1ubq).

For the Gauss model, we perturbed each atom in a random direction by a random amount drawn from a Gaussian distribution with zero mean. We generated different datasets having a standard deviation of $0.5,1.0,2.0$, or 4.0 Å. We also varied the number of structures with $50,100,200,400$, or 800 structures in each data set. In total, we generated ( 3 proteins) $\times$ ( 4 standard deviations) $\times(5$ sizes $)=$ 60 Gaussian datasets.

For the Gō model, we used the SMOG sever [smog] to generate an all-atom Gō model using the default parameters. By construction, the crystal structure is the energetically most favorable structure. Each system was simulated for 5 million time steps using the parameters recommended by the SMOG server at a temperature of $50,70,90$, or 110 reduced units. For each protein at each temperature, we then generated samples of size $50,100,200,400$, or 800 by randomly selecting frames from the 5 million frame full trajectory. In total, we generated ( 3 proteins) $\times$ ( 4 temperatures $) \times(5$ sizes $)=60$ Gō data sets. 


\section{Generation of real-world ensembles}

The purpose of these ensembles is to illustrate how the averaging methods work in ensembles derived from physical models. We examine two cases, which we call Native MD and Refinement.

To generate the Native MD dataset, we took a collection of 14 experimental structures from small proteins (see Supplementary Table 2 for list of structures) using the FF99SB-ILDN parameters [cite], neutralized the proteinsm with sodium or chloride standard ions [cite]parameters and solvated them in TIP3P water [cite]. We simulated them with the "pmemd" program from Amber [cite] using PME, with a Langevin thermostat at $300 \mathrm{~K}$ and constant pressure for $10 \mathrm{~ns}$ ( 2 fs timestep). We used the averaging protocol on the last $5 \mathrm{~ns}$ of simulation sampling every $25 \mathrm{ps}$.

For the Refinement dataset, we used ensembles provided by Mirjalili and Feig, who had the best performing refinement method [ref] in CASP10 [ref]. Briefly, they performed restrained molecular dynamics simulations starting from a non-native structure provided by the CASP organizers. The restraints were either based on information given as "hints" from the CASP organizers or in cases with no hints, based on the assumption that core secondary structure elements are correct. The $\alpha$ carbons of the selected residues were restrained harmonically to their initial coordinates with a force constant of $1 \mathrm{kcal} / \mathrm{mol} / \AA^{2}$. They then selected a subset of conformations based on their DFIRE scores [ref] and the RMSD from the initial template model. For full details on the simulation protocol check reference [ref]. Here, we test our averaging methods on this subset of structures.

\section{Results and Discussion}

\section{NetAvg and CartAvg produce physically reasonable models}

Table 1 shows the MolProbity scores and various related statistics for the experimental structure, the experimental structure after energy minimization, and for NetAvg and CartAvg. Both CartAvg and NetAvg produce physically reasonable models with MolProbity scores of $2.2 \pm 0.5$ and $2.1 \pm 0.5$, respectively. These values are actually lower than for the experimental structures from the PDB. The relatively poor scores for the structures from the PDB can be attributed to the fact that these structures are quite old (published 1987-1992) and significant advances have been made in structure refinement and validation since then.

Minimization of the experimental structure significantly reduces the number of clashes, bad rotameric states, Ramachandran outliers, and overall MolProbity score, which indicates that the force field provides a physical description of the system 
that is reasonably consistent with the statistics from high-resolution crystal structures that are used in MolProbity.

The average structures have worse MolProbity scores than the minimized experimental structure. In particular there are more bad rotamers and bad bond angles, which indicates that the guided minimization at the end of the averaging protocol has introduced some conformational strain into the protein. The structures from CartAvg and NetAvg are not quite at the level of a high-resolution crystal structure, but they display features comparable to crystal structures of $\sim 2.2 \AA$ resolution.

Table 1: MolProbity scores and related statistics for structures from the Gō and Gauss models.

\begin{tabular}{|lllll|}
\hline & Experimental & $\begin{array}{l}\text { Minimized } \\
\text { Experimental }^{2}\end{array}$ & $\begin{array}{l}\text { After } \\
\text { CartAvg }^{-}\end{array}$ & $\begin{array}{l}\text { After } \\
\text { NetAvg }\end{array}$ \\
\hline Clash Score $^{3}$ & 23.0 & 5.5 & 5.6 & 5.5 \\
\hline Bad Rotamers & $15.6 \%$ & $1.1 \%$ & $6.6 \%$ & $6.5 \%$ \\
\hline $\begin{array}{l}\text { Ramachandran } \\
\text { Outliers }\end{array}$ & $2.9 \%$ & $1.1 \%$ & $1.9 \%$ & $1.5 \%$ \\
\hline CB Deviation & $0.4 \%$ & $0.6 \%$ & $0.6 \%$ & $0.8 \%$ \\
\hline Bad bond lengths & $0 \%$ & $0 \%$ & $0 \%$ & $0 \%$ \\
\hline Bad bond angles & $1.3 \%$ & $0 \%$ & $1.4 \%$ & $4.2 \%$ \\
\hline MPscore & 3.1 & 1.6 & 2.2 & 2.1 \\
\hline
\end{tabular}

${ }^{1}$ Average scores for PDB entries 1bdd, 2gb1, 1ubq

${ }^{2}$ Average scores for PDB entries 1bdd, 2gb1, 1ubq after energy minimization with the same protocol used for NetAvg and CartAvg

${ }^{3}$ Bad steric clashes per 1000 atoms

${ }^{4}$ MPscore indicates the model has similar characteristics to a crystal structure of this resolution. Lower is better. 


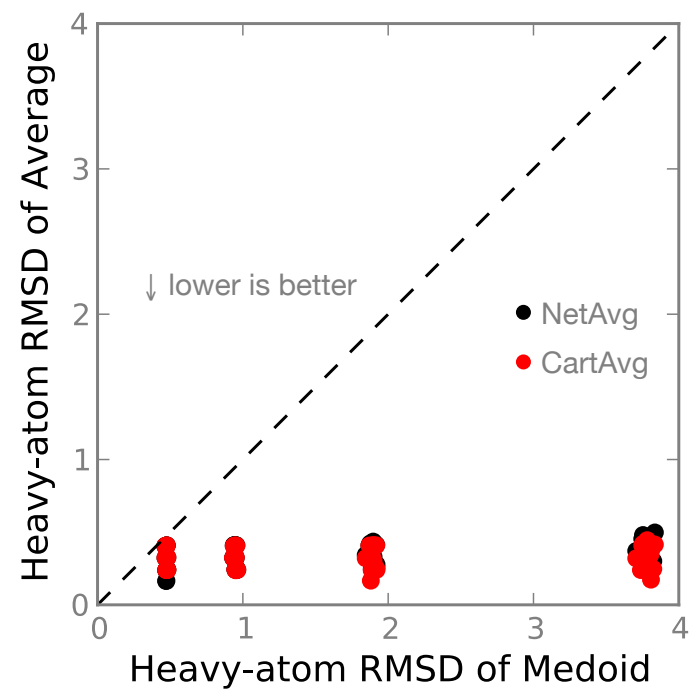

Figure 4: Both CartAvg and NetAvg systematically improve over the medoid structure on the Gaussian model systems.

The Gauss model is primarily intended as a control. There are no couplings between atoms and the distribution of each atom is a simple isotropic Gaussian, so we expect the averaging protocols to work well. Indeed, both CartAvg and NetAvg perform well on this test (Figure 4), and give dramatic improvements in RMSD compared to the medoid model of the ensemble. On average, CartAvg performs slightly better than NetAvg. This difference is statistically significant (Supplementary Table 1), but small $(0.02 \AA)$.

The final quality of the model is independent of the quality of the medoid. This is expected as even when the cloud of points for each atom is very broad (leading to a high medoid RMSD), we can still accurately estimate the center of the ensemble provided we have a modest number of samples.

NetAvg outperforms the other methods on samples from the Gō model 

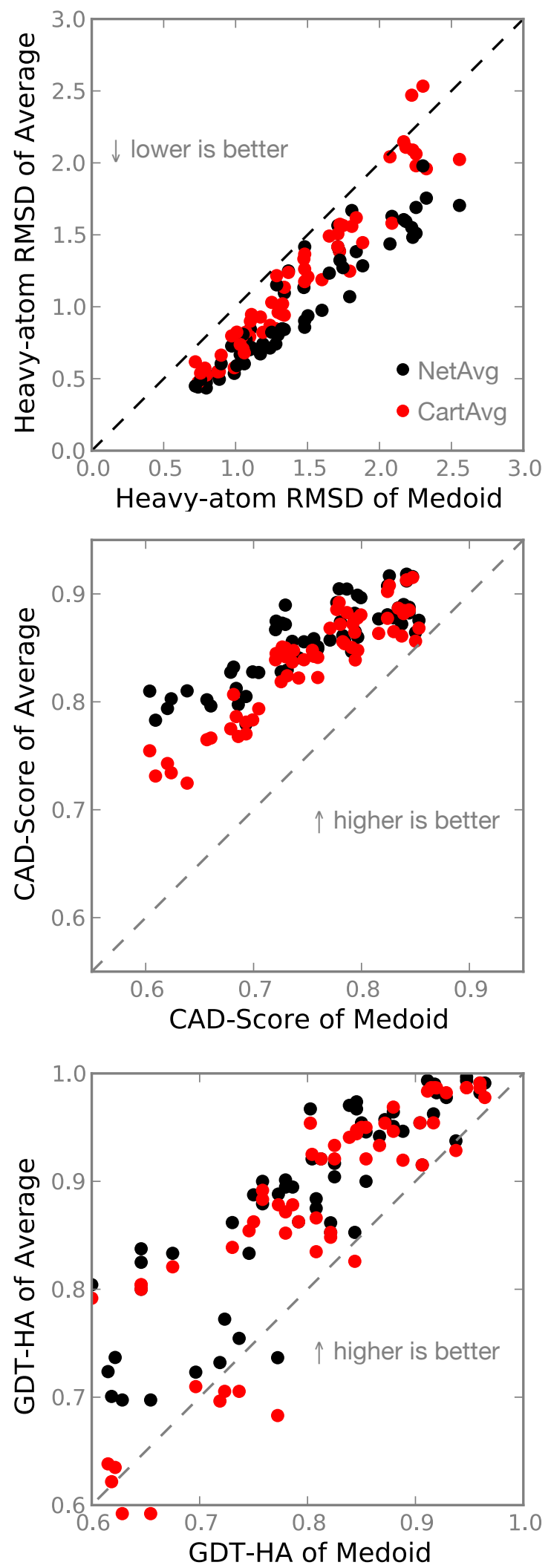

Figure 5: Both NetAvg and CartAvg improve over the medoid structure on the Gō model systems. Overall, NetAvg performs better than CartAvg. 
Figure 5 shows that in almost all cases, both NetAvg and CartAvg improve over the medoid structure of the ensemble according to a variety of measures. Table 2 summarizes the degree of improvement for each score with both averaging methods. For all measures, the NetAvg and CartAvg models are better than the medoid and the differences are statistically significant (Supplementary Table 1). In all cases, NetAvg is significantly better than CartAvg.

Table 2: Improvement of average structure relative to medoid structure from ensemble

\begin{tabular}{|lrr|}
\hline & NetAvg & \multicolumn{1}{c|}{ CartAvg } \\
\hline Average RMSD improvement $(\AA)$ & 0.42 & 0.25 \\
\hline Average percent RMSD improvement & 31 & 20 \\
\hline Average GDT-HA improvement & 0.08 & 0.05 \\
\hline Average percent GDT-HA improvement & 11 & 8 \\
\hline Average CAD-score improvement & 0.10 & 0.08 \\
\hline Average percent CAD-score improvement & 14 & 11 \\
\hline
\end{tabular}

The Gō model can produce complex, curved distributions of the type shown in Figure 2. NetAvg was designed explicitly to deal with such distributions and, as expected, performs better than CartAvg on this datset. 

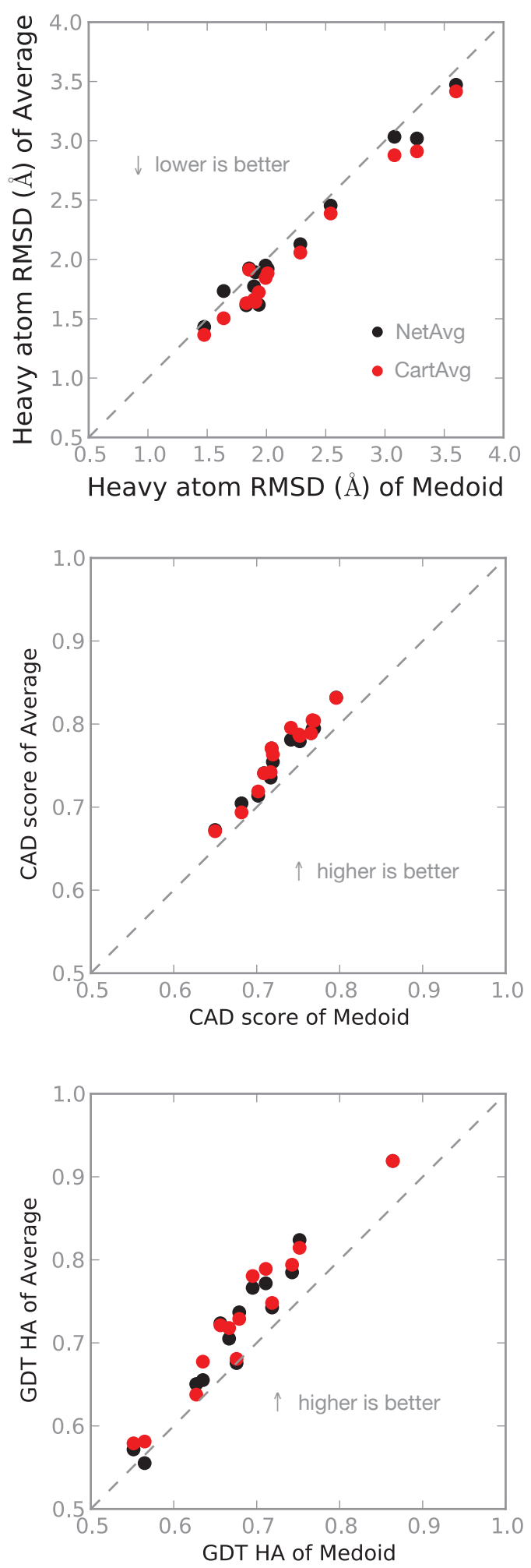

Figure 6: Both NetAvg and CartAvg improve over the medoid structure on the Native MD dataset. 
Figure 6 shows that on average both NetAvg and CartAvg produce models that are closer to the crystal structure than the medoid of the ensemble for the Native MD dataset. Table 3 shows that the mean improvements range from 4.0 to 8.5 percent, depending on the method and the score being assessed. Both NetAvg and CartAvg are significantly better than the medoid, but the difference between NetAvg and CartAvg is not significant for any of the three scores (RMSD, GDT-HA, CAD-score; Supplementary Table 1).

Table 3: Improvement of average structure relative to the medoid for the native centered structures.

\begin{tabular}{|lrr|}
\hline & NetAvg & CartAvg \\
\hline Average RMSD improvement $(\AA)$ & 0.10 & 0.18 \\
\hline Average percent RMSD improvement & 4.2 & 8.5 \\
\hline Average GDT-HA improvement & 0.04 & 0.05 \\
\hline Average percent GDT-HA improvement & 5.5 & 6.5 \\
\hline Average CAD-score improvement & 0.03 & 0.03 \\
\hline Average percent CAD-score improvement & 4.0 & 4.0 \\
\hline
\end{tabular}

It is obvious that the performance on the Native MD dataset is not nearly as good as either of the ideal datasets (Gō, Gauss). We will return to this point later.

\section{NetAvg and CartAvg give small improvements on the Refinement dataset}

This dataset is based on restrained molecular dynamics simulations with a relatively stiff force constant of $1 \mathrm{kcal} /\left(\mathrm{mol} \AA^{2}\right)$ enforced on the backbone, which results in very "tight" ensembles, with small conformational fluctuations (Figure 7). Both NetAvg and CartAvg make small improvements to the RMSD, GDT-HA, and CADscore over the medoid (Figure 8 and Table 4), although some of the results are not statistically significant: CAD-score for CartAvg, RMSD for NetAvg, and the differences between NetAvg and CartAvg for all three scores (Supplementary Table 1).

Table 4: Improvement of average structure relative to the medoid for the Refinement dataset

\begin{tabular}{|lll|}
\hline & NetAvg & CartAvg \\
\hline Average RMSD improvement $(\AA)$ & 0.10 & 0.12 \\
\hline Average percent RMSD improvement & 2.3 & 3.0 \\
\hline Average GDT-HA improvement & 0.01 & 0.02 \\
\hline Average percent GDT-HA improvement & 2.5 & 2.6 \\
\hline Average CAD-score improvement & 0.01 & 0.02 \\
\hline Average percent CAD-score improvement & 1.5 & 2.5 \\
\hline
\end{tabular}




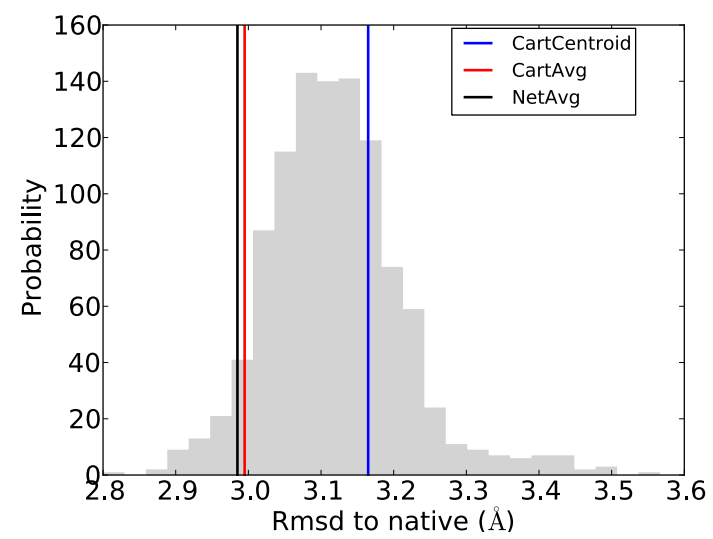

Figure 7. Representative RMSD distribution from the Refinement dataset (PDB 3DB5, from CASP8). The heavy atom RMSD of the ensemble is shown in grey. Vertical lines denote RMSD of a single structure. The NetAvg and CartAvg structures are better than the medoid and better than most of the structures in the ensemble. 


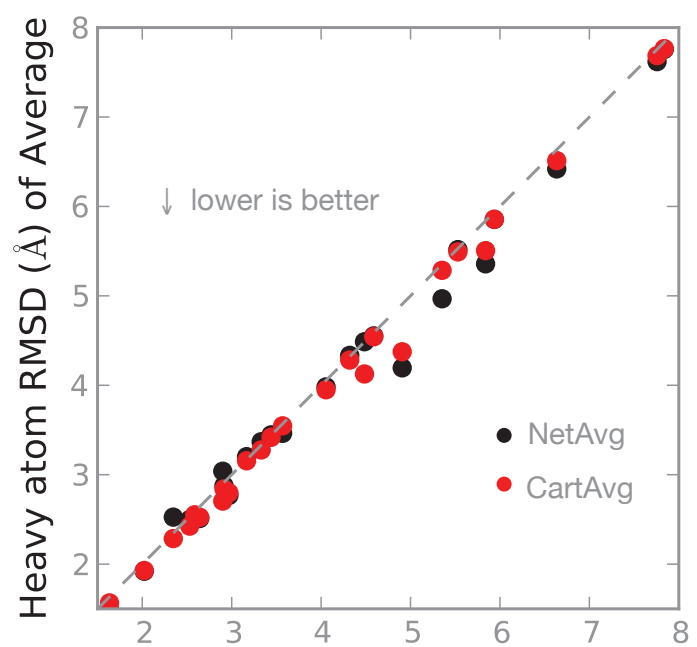

Heavy atom RMSD ( $\AA$ ) of Medoid
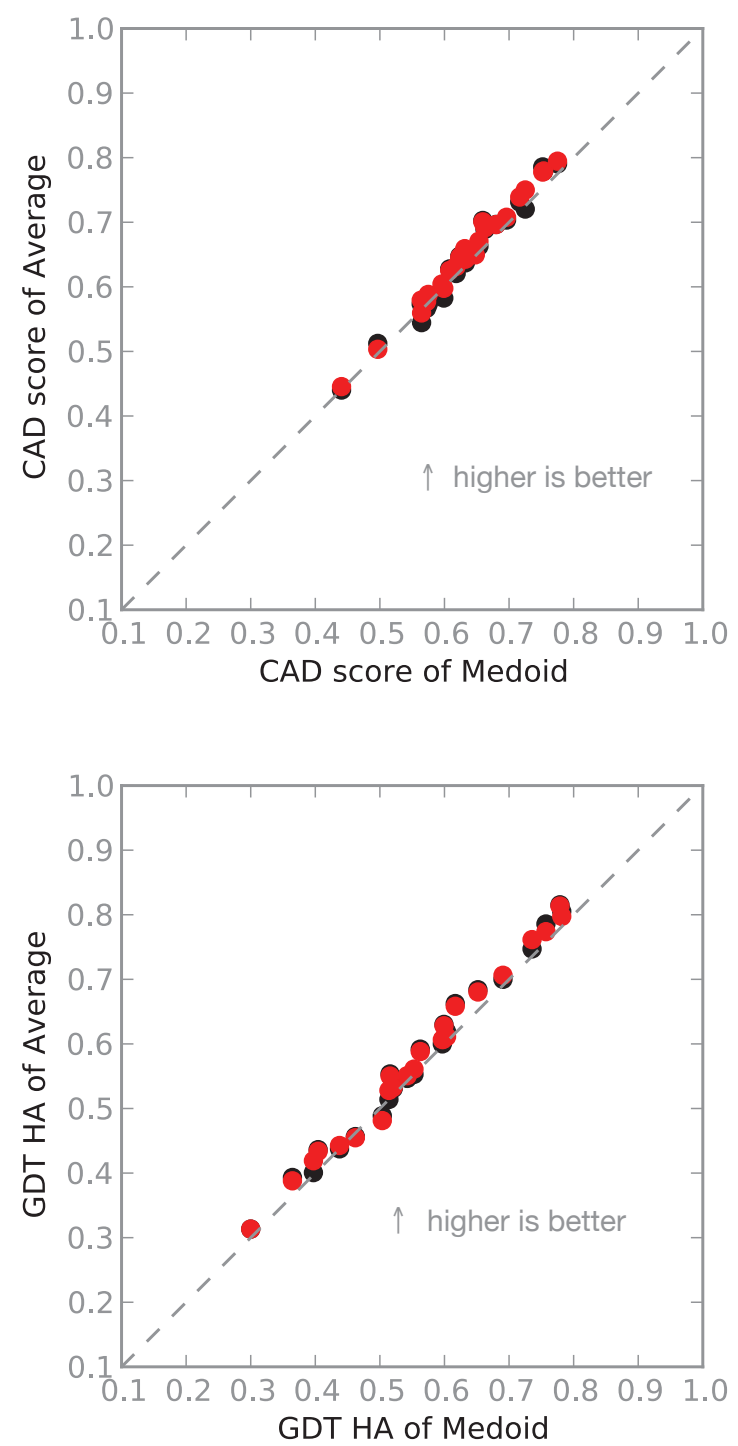
Figure 8. NetAvg and CartAvg give slight improvements on the Refinement dataset.

The RMSD distributions are usually tightly clustered due to the use of strong restraints (Figure 7). Although the averaging methods offer only small improvements over the medoid, they are often better than a majority of the structures in the initial ensemble.

The averaging methods display their worst overall performance on this dataset. We will return to this point later.

\section{The performance of averaging is related to the width of the conformational ensemble}

When the conformational ensemble is very "tight", there is relatively little that averaging can do to improve models. All of the structures are very similar and the average-which lies within the cloud of structures-must also be similar. In contrast, averaging can improve more when the ensemble is broad, because there is more to gain by choosing the correct position for each atom. This is evident in all of the datasets, particularly the Gauss (Figure 4) and Gō (Figure 5) datasets.

This also helps to explain why the performance on the Refinement dataset was worse than other cases. The refinement simulations were conducted with relatively stiff position restraints, which prevent major fluctuations in conformation, which is evident in Figure 7 and Figure 9. With such tight ensembles, it is difficult for any of the averaging methods to improve much over the medoid.

We also wondered about the possibility of outliers in the dataset to influence the average structure. These are structures that are very different from the rest of the ensemble and produce a significant deviation in the average structure. We devised a 'pruning algorithm', described in SI to reduce the effect of these outliers. In our experience with our datasets pruning has little effect on the resulting average structure (see SI Figure 1).

\section{For averaging to be successful, the atom cloud must overlap with the atom position in the native structure}

The idea of averaging, regardless of the method used, is to select the most representative position for each atom from within the space defined by the ensemble. These methods are not capable of recovering an average position that lies outside of the cloud of positions sampled for each atom (Figure 9). The implication of this is that if there is no overlap between the ensemble and the native state, averaging will not be able to correct this. 


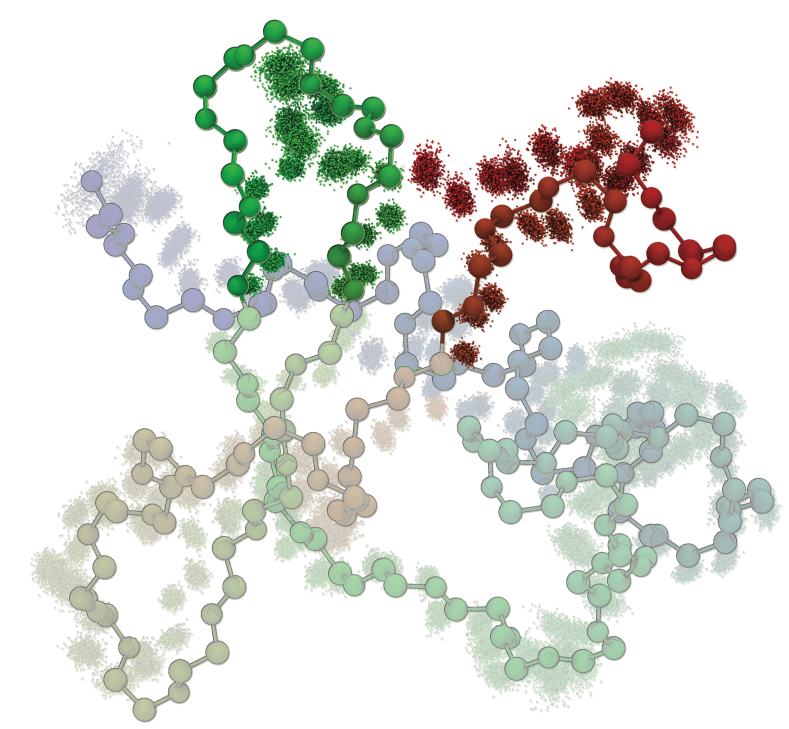

Figure 9: Successful averaging requires overlap between the sampled ensemble and the experimental structure. This panel shows the lack overlap between the simulated ensemble and experimental structure for one of the proteins (PDB ID: 2k5r) from the Refinement dataset. Positions for the N, C, CA atoms are shown as spheres for the experimental structure and clouds of points for the refinement ensemble. The coloring goes from blue ( $\mathrm{N}$-termini) to red (C-termini). Highlighted are a loop region (green) and the C-termini (red) for which the overlap between the ensemble and the experimental structure is poor.

The results on the two ideal datasets (Gō and Gauss, Figures 4 and 5) are substantially better than on the two real-world datasets (Native MD and Refinement, Figures 6 and 8). The ideal datasets have been constructed so that the experimental structure is at the center of the ensemble, while the real-world datasets are subject to bias and errors in the force field. Additionally, for refinement, the simulations are not started from the experimental structure.

We can quantify the degree of systematic bias by using our knowledge of the experimental structure to construct a hybrid best model, where, for each atom, we take the position from the sampled ensemble that is closest to correct. If the RMSD of this hybrid best model is low, it means that each atom samples a position close to the correct position at least some of the time. If the RMSD is high, it means that there are atoms that never sample positions close to the correct position.

The average RMSD of the hybrid best models is much higher for the two real-world datasets (Figure 10), which indicates that some atoms in the real-world dataset are systematically shifted away from the correct position. There is no way that any of the averaging schemes can recover from this. 


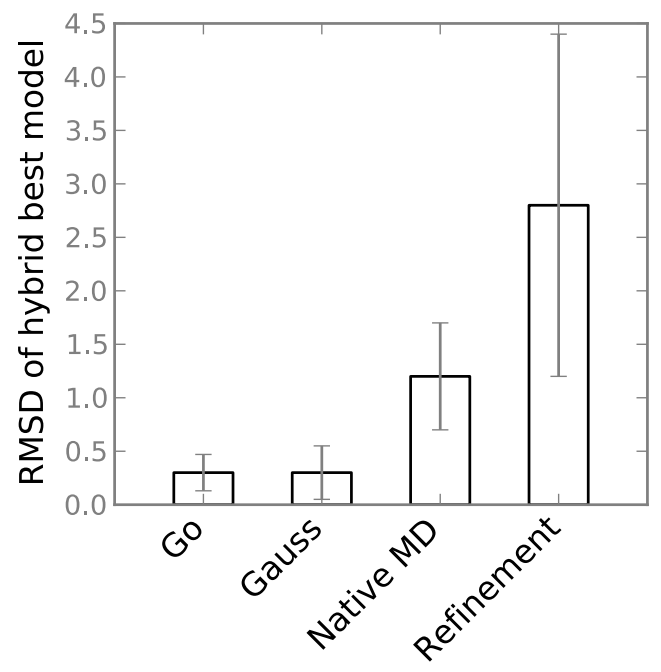

Figure 10: The Native MD and Refinement datasets contain atoms that do not sample positions near the experimentally observed positions. This panel shows the average heavy atom RMSD for the hybrid best models for each dataset (see text for details).

\section{Rules of thumb for selecting average structures from ensembles}

We now give a few rules of thumb for selecting average structures from conformational ensembles.

- One should routinely employ some kind of averaging procedure. This usually improves the structures by some amount, produces physically realistic structures, and is computationally cheap.

- An argument can be made for only using structures that were actually sampled (such as the medoid). However, as Figure 1 shows, entropy will always push you towards a higher RMSD, even though there are other structures that have lower energy and are a better representation of the ensemble.

- If the ensemble is "tight", neither CartAvg or NetAvg can improve much over the medoid

- For ensembles where some atoms have curved distributions, prefer NetAvg over CartAvg. For other ensembles, there is little difference between the methods.

- For large datasets (over 1000 structures), prefer CartAvg as the eigenvector centrality calculation in NetAvg becomes expensive.

- It is essential to use some kind of minimization procedure, as the raw average structure will contain significant physical defects because correlations between atoms have been ignored. 


\section{Conclusions}

We have presented here two very computationally inexpensive methods (CartAvg and NetAvg) that on provide structures that are better representations of the ensemble than the standard practice of using the medoid. NetAvg is designed to deal with curved distributions of atoms and performs well on an ideal dataset. In practice, the effectiveness of both methods is often hindered by errors in the underlying atom distributions. We expect these protocols to be easily adapted in to pipelines in which large ensembles of structures are produced (e.g. structure prediction or docking studies).

\section{Supplementary Information}

Supplementary Table 1: This table reports if the differences observed between averaging methods are significant, as judged by the two-sided sign test with a p-value of 0.01 . A '+' indicates a result is significant. A '-' indicates it is not. For each entry, the first symbol indicates RMSD, the second indicates GDT-HA, and the third indicates CAD-score.

\begin{tabular}{|l|l|l|l|l|}
\hline & Gō & Gauss $^{1}$ & $\begin{array}{l}\text { Native } \\
\text { MD }\end{array}$ & Refinement \\
\hline $\begin{array}{l}\text { CartAvg } \\
\text { vs } \\
\text { Centroid }\end{array}$ & +++ & + & +++ & ++- \\
\hline $\begin{array}{l}\text { NetAvg } \\
\text { vs } \\
\text { Centroid }\end{array}$ & +++ & + & +++ & -++ \\
\hline $\begin{array}{l}\text { CartAvg } \\
\text { vs } \\
\text { NetAvg }\end{array}$ & +++ & + & -- & -- \\
\hline
\end{tabular}

${ }^{1}$ For Gauss models, only RMSD was compared

Supplementary Table 2: Experimental structures used to generate the Native MD dataset.

\begin{tabular}{|l|l|}
\hline PDB ID & PDB ID \\
\hline 3pnx & $3 \mathrm{n} 6 \mathrm{y}$ \\
\hline $3 \mathrm{npp}$ & $3 \mathrm{nhv}$ \\
\hline $2 \mathrm{kyy}$ & $3 \mathrm{ni} 8$ \\
\hline $3 \mathrm{n} 70$ & $3 \mathrm{noh}$ \\
\hline $2 \mathrm{kyw}$ & $3 \mathrm{voq}$ \\
\hline $3 \mathrm{nrf}$ & $3 \mathrm{nkl}$ \\
\hline 3na2 & 3nrl \\
\hline
\end{tabular}




\section{Methods}

\section{Pruning Protocol}

Given an ensemble, can we produce a more robust description of it by reducing the number of outlier structures? To answer this question we perform a two stage protocol: (A) we first cluster our ensemble by using self-similarity (measured by RMSD) and (B) remove structures from the cluster that are outliers. For the clustering we use standard clustering protocols such as the ones implemented in ptraj [cite]. For the pruning step we have to define 3 parameters:

- Dimension for Multidimensional Scaling (MDS) projection to low dimensional space.

- Trim factor (trim): either a multiplication factor (for $\operatorname{dim}>1$ ) or $\%$ of points you want to remove (for $\operatorname{dim}=1$ ).

- NN: number of neighbors that need to occur in neighborhood of point in the low-dimensional space for the point to be retained in the cluster (only used for $\operatorname{dim}>1$ ).

For each cluster, the distance matrix for the subset of observations in the cluster is extracted and used to project the points onto a lower dimension space using MDS. For projections to 1D, the trim factor is used to prune a percentage of points from each end of the projection. For example, a trim factor of 10 would prune 5 percent of the observations at each end based on their coordinates in the projected space. For projections to 2D or higher, the distance matrix for the subset of observations in the cluster is computed, the points projected down, and the standard deviation of all the pairwise distances for points within the cluster is computed (SD). The trim factor (trim) is multiplied by SD, and an epsilon ball with epsilon = trim*SD is placed around each point in the low dimension space. Points must have NN neighbors within their trim*SD ball or they are pruned out of the cluster (where NN is a parameter specifying the threshold number of neighbors to keep a point in the dataset). The algorithm returns the subset of the original cluster that was retained after pruning. From this subset we can then use both methods described in the main text to average and minimize; we call this new methods with pruning PNetAvg and PCartAvg. Figure SI 1 shows the effect pruning had in the most populated clusters of a subset of five proteins from CASP 11 (PDB IDs:4fr9, 4fj6, 4epz, and CASP IDs: T0709, Tr750 (not yet released in PDB)). Despite our limited success with the pruning algorithm, we feel there might be some value in exploring more options in the future. 


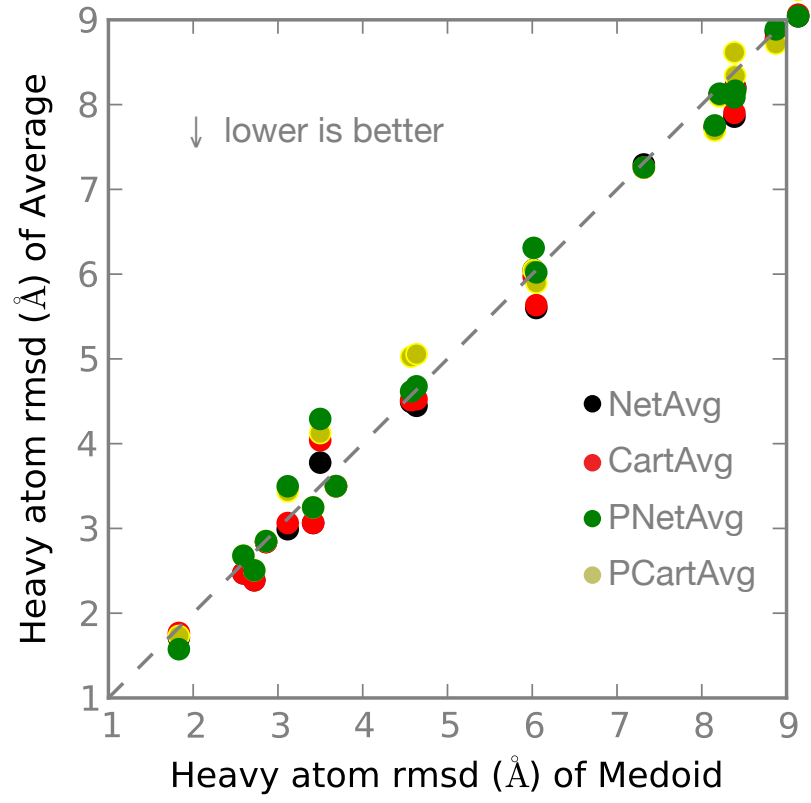

Figure S1. Both PCartAvg and PNetAvg show no clear improvement over the NetAvg and CartAvg methods previously described. 